\title{
Performance Analysis of Suboptimal Spectrum Sensing Algorithm in Cognitive Networks
}

\author{
D. T. Do \\ Department of Communications Engineering, School of Electrical and Electronics Engineering, Ton Duc Thang University, Ho Chi \\ Minh City, Vietnam
}

\begin{abstract}
Recently, both optimal and suboptimal spectrum sensing approaches have attracted extensive attention in wireless communications field. Thanks to opportunistic spectrum access schemes, different cognitive users can cooperatively search for and exploit instantaneous spectrum availability. In this paper, we address the analysis of spectrum sensing in opportunistic spectrum access models and then determine throughput of the cognitive radio system. Our goal in all designs is reach optimal access policy for each channel with the high cooperative sensing performances.
\end{abstract}

Keywords Cognitive Radio, Spectrum Sensing

\section{Introduction}

It is well-known that the current spectrum management policy has resulted in an under-utilized spectrum[1]. In order to enhance limited spectrum resource, cognitive radio (CR) network is proposed. Especially, cognitive radio is considered as a key technology to deal with this increasingly tense situation in spectrum use. It enables public access to those unused spectral range by allowing the unlicensed (cognitive) users to opportunistically access to the licensed spectrum, and therefore the overall spectral efficiency can be economically increased. Basically, the secondary user (SU) to search for and utilize instantaneous spectrum opportunities left by primary user (PU), while limiting its interference to PU. Related to the spectrum opportunities, the SU should first model PU's behavior. In the previous literature, they proposed two models, namely, discrete-time and continuous-time models. In discrete-time model, both PU and SU are time-slotted. It can be seen that, in[1], an opportunistic MAC protocol with random and negotiation-based sensing for ad-hoc networks is proposed. With the optimal spectrum sensing and access strategies, we often concern that the synchronization issues of all PUs and SUs in discrete-time model is necessary. In fact, it causes more overhead and time offset may be fatal for SU's MAC strategy. In[2, 3], the optimal sensing period is derived for the simplest single-channel model.

Methodology is designed to sense the spectrum in a relia

*Corresponding author:

dodinhthuan@gmail.com (D. T. Do)

Published online at http://journal.sapub.org/ajsp

Copyright (C) 2011 Scientific \& Academic Publishing. All Rights Reserved ble manner becomes one of the major challenges in implementation of the cognitive radio. Due to complex channel fading and shadowing effects, the received signal strength at the cognitive user may be severely degraded, and the sensing performance of the single detector is limited. As a result, cooperative spectrum sensing approaches, which can enhance the sensing performance, have attracted extensive attention. In literature, a number of opportunistic spectrum access strategies have been investigated for cognitive network. Most of them are based on the assumption that each of the cognitive users has the full spectrum sensing ability for wide spectrum band. In real condition, due to hardware limitation, the cost to achieve wide-band spectrum sensing by one single cognitive user is quite high $[4,5]$. And it is realistic that a cognitive user is able to sense limited bandwidth of spectrum during a certain amount of time.

Medium Access Control has an important role in several cognitive radio functions: spectrum mobility, channel sensing, resource allocation, and spectrum sharing[3]. Spectrum mo- ability allows a secondary user to vacate its channel, when a primary user is detected, and to access an idle band where it can reestablish the communication link. Channel sensing is the ability of a cognitive user to collect information about spectrum usage, and to maintain a dynamic picture of available channels. Resource allocation is employed to opportunistically assign available channels to cognitive users according to QoS requests. Spectrum access deals with contentions between heterogeneous primary and secondary users in order to avoid harmful interference. Multi-channel MAC protocols for ad-hoc wireless networks have represented a first step in the development of MAC protocols for cognitive radio in unlicensed scenarios. These protocols address similar problems; they operate in a multi-channel 
context, and face the multiple channel hidden terminal problems[4]. A cognitive radio may exploit, however, increased sophisticated sensing functionalities; it distinguishes between primary and secondary users, and provides protection to licensed transmissions. The number of channels available at each user is fixed in a multi-channel network, while it varies with time and space in a cognitive network. Furthermore, the time-scale in which a cognitive radio operates is very different from that of an ad-hoc radio: secondary users must exploit periodical sensing to be aware of the wireless environment evolution, and must rapidly adapt their behaviour to reach QoS.

In this paper, we mainly contribute to design of Medium Access Control (MAC) layer which has an important role in several cognitive radio functions: spectrum mobility, channel sensing, spectrum resource allocation, and spectrum sharing[6]. We can analyze these procedures carefully. Firstly, spectrum mobility allows a SU to vacate its channel, when a PU is detected, and to access an idle band where can reestablish the communication link. An then channel sensing procedure help cognitive user to collect information about spectrum usage, and to maintain a dynamic picture of available channels. While resource allocation is employed to opportunistically assign available channels to cognitive users according to QoS requests. Interestingly, spectrum access approaches deals with contentions between heterogeneous primary and secondary users in order to avoid harmful interference.

In the cognitive communications, Multi-channel MAC protocols for ad-hoc wireless networks have represented a early development of MAC protocols for cognitive radio in unlicensed scenarios. Unfortunately, these protocols address similar problems; they operate as multichannel systems, and face the multiple channel hidden terminal problems[6-8]. It is true that a cognitive radio may increase sophisticated sensing functionalities; it distinguishes between PUs and SUs, and provides protection to licensed transmissions. The number of channels available at each user is fixed in a multi-channel network, while it varies with time and space in a cognitive network. Furthermore, the time-scale in which a cognitive radio operates is very different from that of an ad-hoc radio: secondary users must exploit periodical sensing to be aware of the wireless environment evolution, and must rapidly adapt their behavior to reach QoS and comply with interference constraints.

The rest of the paper is organized as follows. In section II, we introduce the system model for MAC layer design in Opportunistic Spectrum Access requirement. Next, we analyze the optimal spectrum sensing scheme in different scenarios in section III. The numerical results are presented in section IV and the concluding remarks are pointed out in section $\mathrm{V}$.

\section{System Model}

We consider a spectrum consisting of $M$ channels in this paper. Each channel has the bandwidth $B_{i}(i=1, \ldots, N)$. These $\mathrm{N}$ channels are licensed to a primary network whose users operate following the synchronous slot structure. The traffic of all of the PUs are such that the occupancy of these $\mathrm{N}$ channels obeys a discrete-time Markov chain with $M=2^{N}$ states. The network state in the slot $t$ is given by $\left[S_{1}(t), \ldots, S_{N}(t)\right]$ where $S_{i}(t)$ is a set of $\{0$ (occupied $), 1($ idle $)\}$. Assuming that the spectrum usage statistics of the PUs' network remain unchanged during $\mathrm{T}$ slots. In fact, this spectrum is licensed to a primary network, whose users are operated in a synchronous time-slotted fashion. Since the primary network does not use the whole spectrum all the time, it is assumed that the probability of channel $i$ being occupied by the primary network in one time slot is $\theta_{i}\left(0 \leq \theta_{i} \leq 1, i=1, \ldots, M\right)$. These values are affected by the channel allocation schemes and traffic statistics of the primary network.

Besides, our duties focus on maximizing SU's total channel utilization while limiting its interference to the PUs. We also model the interference between PU and SU via the average temporal overlap. The formulation describes the interference $I_{i}$ between $\mathrm{SU}$ and $\mathrm{PU}_{\mathrm{i}}$ is

$$
I_{i}=\lim _{T \rightarrow \infty} \frac{\int_{0}^{T} 1\left\{A_{i}(\tau) \cap B_{i}(\tau)\right\} d \tau}{T}
$$

Where $1\{$.$\} is the indicator function of the event en-$ closed in the brackets; $A(\tau)$ and $B_{i}(\tau)$ denote the event that $\mathrm{PU}_{\mathrm{i}}$ and $\mathrm{SU}$ access channel $i$ at time $\tau$, respectively.

The channel utilization is defined as the SU's temporal utilization ratio (i.e., the transmission time divided by the total time). These SU's channel utilization $U_{i}$ on channel $i$ is

$$
U_{i}=\lim _{T \rightarrow \infty} \frac{\int_{0}^{T} 1\left\{B_{i}(\tau)\right\} d \tau}{T}
$$

\section{The Suboptimal Strategy for Spec- trum Sensing}

Spectrum sensing is the functionality enabling cognitive radios to be aware of spectrum usage and to detect spectrum opportunities. When two nodes want to communicate, source and destination are responsible for performing sensing; they select a set of channels to sense, they estimate channel availability, then channel filtering is performed, and a communication link is set up. Both reactive (on-demand) and proactive sensing may be exploited in a cognitive network. During data transmission, periodic in-band sensing is performed to detect incumbent PUs and avoid harmful collisions, while the sensing process dealing with the search of new opportunistic resources is referred to as out-of-band sensing. In this framework, we do not concern about techniques processing observations and detect PUs. 
There are two kind of the sensing outcome processing and available channel estimation, namely a distributed and centralized fashion. In the centralized approach, a leader fuses all sensing information according to a certain rule (for example, AND, OR, or M-out-of-N rules) and it evaluates spectrum opportunities. In the distributed solution, secondary nodes share observation data and independently take decisions regarding resource availability[4-12].

In the cognitive radio technology, the optimal spectrum sensing policy can be computationally prohibilitive. The reason here is that the dimension of the sufficient statistic grows exponentially with the number of channel N. In fact, the optimal algorithm can be computed offline and process prior to the SU want to access the required spectrum. This approach makes it difficult to adapt to changes in the spectrum occupancy statistics. This is motivation for develop suboptimal spectrum sensing schemes. Let $\Omega=[\omega, \ldots, \omega]$ where $\omega$ is the probability that channel $i$ is available at the beginning of a slot. As a result, the dimension of the sufficient statistic reduces from $2^{N}$ to $\mathrm{N}$. This result points to the possibility of significantly reducing the computational complexity of the optimal Opportunistic Spectrum Access issues. Through the sufficient statistic, we apply the suboptimal scheme based on a greedy approach that maximizes per slot throughput. The analyzed systems have independently channels.

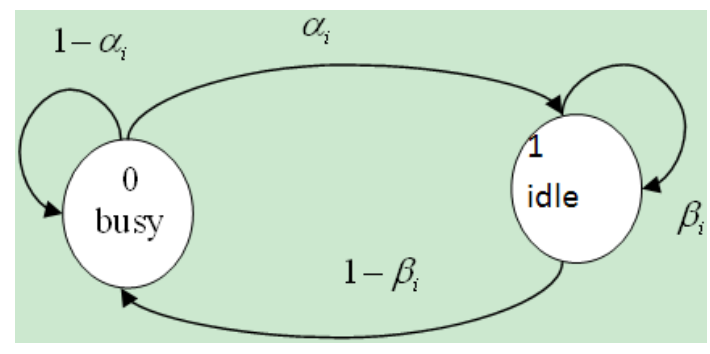

Figure 1. The Markov chain diagram.

As the Figure 1, we can see clearly that channel $i$ transits from the state 0 to state 1 with the probability $\alpha_{i}$ and stays in the state 1 with the probability of $\beta_{i}$. We gain the expected reward in the slot $t$ if channel $\mathrm{c}$ is selected as follow

$$
B_{c}\left(\omega_{c}(t) \beta_{c}+\left(1-\omega_{c}(t)\right) \alpha_{c}\right)
$$

Where $\omega_{c}(t) \beta_{c}+\left(1-\omega_{c}(t)\right) \alpha_{c}$ is the probability that channel $c$ will be available in the slot $t$. Applying the greedy approach, the action in slot $t$ is chosen aiming to maximize the immediate reward below

$$
c_{\text {max }}(t)=\arg \max _{c=1, \ldots, N} B_{c}\left(\omega_{c}(t) \beta_{c}+\left(1-\omega_{c}(t)\right) \alpha_{c}\right)
$$

In next slot, we have the formula

$$
\Omega(t+1)=\left[\omega_{1}(t+1), \ldots, \omega_{N}(t+1)\right]=\Im\left(\Omega(t) \mid c_{\max }(t), \Theta_{c \max }(t)\right)
$$

Where the belief vector $\Omega$ is updated based on the action $c_{\max }(t)$ and the observation at the end of the slot $t$; $\Im($.$) function represents the updated knowledge of the net-$ work state after incorporating the action and observation obtained in selected slot[13-18].
Note that when the channel is not sensed, the probability of its availability is updated according to the Markov chain. But channel is sensed will be lead to the belief vector records the channel state prior to the state transition at the beginning of each slot.

\section{Simulation Results}

In simulations, we present numerical results to evaluate the performance of the suboptimal cognitive MAC schemes. We consider some independent channels with the same bandwidth $B=1$ and transition probabilities $\{\alpha, \beta\}$.

In the first experiment, we set up 4 independent channel for determining the throughput. If we increase the alpha probability parameter then its throughput degraded seriously.

Next, we present the different number of the sensed channels in Figure 3. We can see the more channel based spectrum sensing method has better performance compared to another. Consequently, through this illustration we gain optimal performance together with design of the communication links between the source nodes and destination nodes properly. This result confirms the chosen suboptimal spectrum sensing methodology will be reduced the complexity in computational functions.

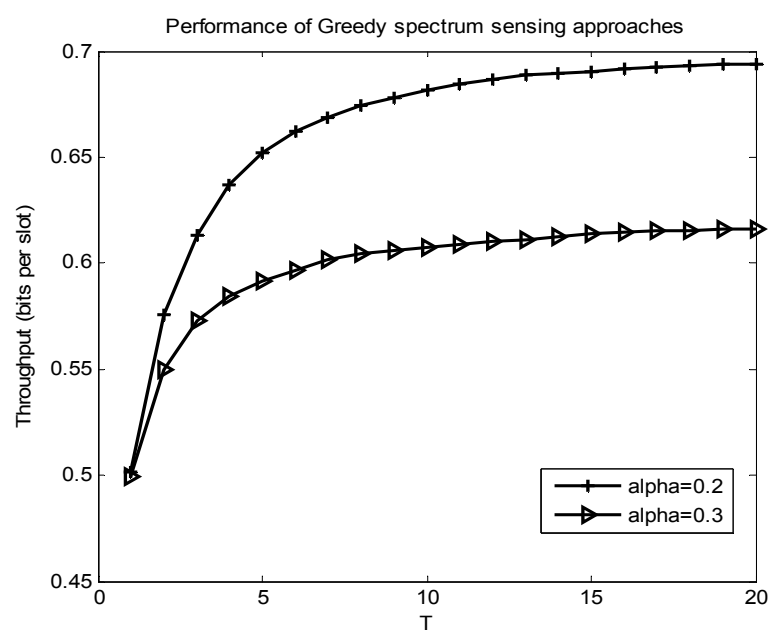

Figure 2. Throughput analysis of the Greedy approach

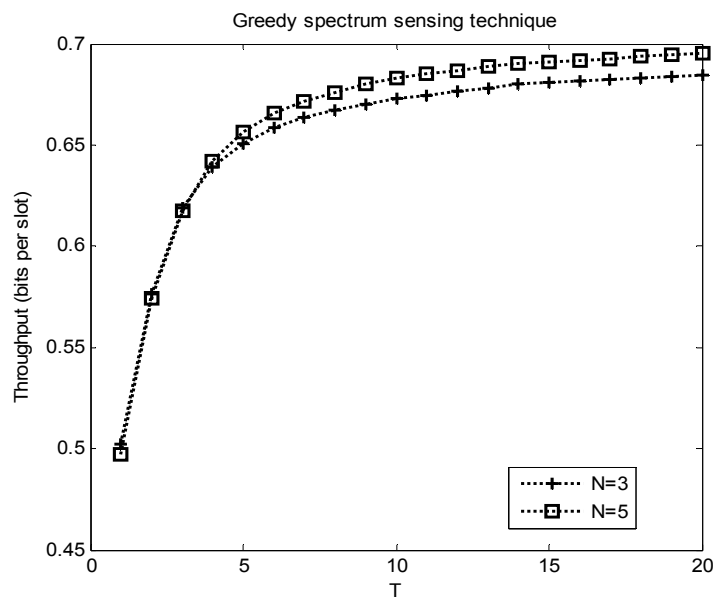

Figure 3. The performance comparison with different schemes. 


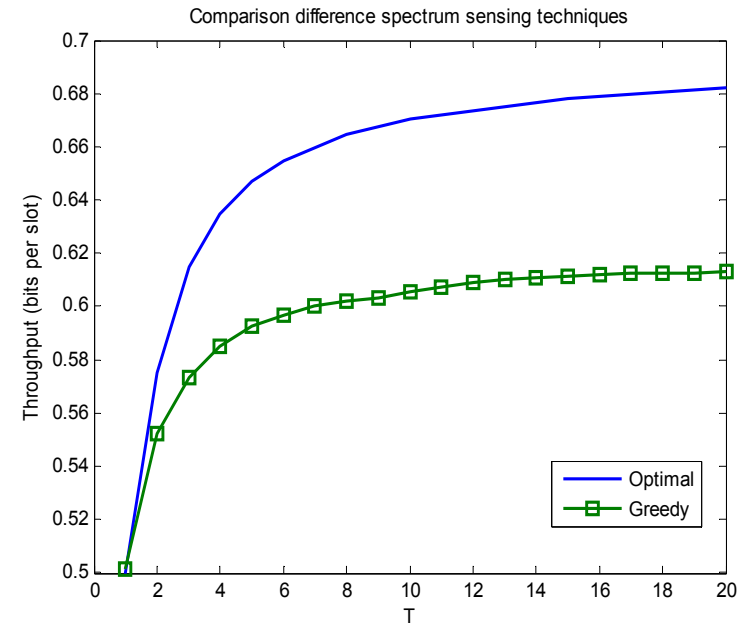

Figure 4. The optimal and Greedy schemes.

In Figure 4, we compare the throughput per slot of the suboptimal model with optimal one. In this simulation, we set up the number of independent channel of 4 and alpha index probability is 0.3 . It can be seen that the suboptimal performance for spectrum sensing is acceptable in real applications.

\section{Conclusions}

We have presented the performance of throughput of the suboptimal spectrum sensing technique and optimal one as well. Throughout the paper, it can be seen that the selection of alpha parameter of the probability in the selected node at value of 0.2 bring the good performance for cognitive radio. We try to change some determined parameters aiming to prove that the cognitive radio can implementation in reality with proper number of independent channel and the state transition probability. The throughput analyses in the simulations are clear demonstrations providing the improvement work for cognitive networks, especially in opportunistic spectrum access schemes.

\section{REFERENCES}

[1] H. Su and X. Zhang, "Cross-layer based opportunistic MAC protocols for QoS provisionings over cognitive radio mobile wireless networks," IEEE J. Sel. Areas Commun., vol. 26, no. 1, pp. 118-129, Jan. 2008

[2] Y. Pei, A. T. Hoang, and Y. Liang, "Sensing-throughput tradeoff in cognitive radio networks: How frequently should spectrum sensing be carried out?" in Proc. of 18th IEEE PIMRC, Athens, Greece, Sep. 2007

[3] Y. Xu, Y. Li, H. Zou, X. Yang, "Joint sensing period optimization and transmission time allocation for cognitive radio networks," in Proc. of the WCSP 2009, Nanjing, China, Nov. 2009

[4] Juncheng Jia, Qian Zhang and Xuemin Shen, "HC-MAC: A Hardware-Constrained Cognitive MAC for Effcient Spectrum Management," IEEE Journal on Selected Areas in
Communications, Vol 26, Issue 1, pp. 106 - 117, Jan. 2008

[5] Yunxia Chen; Qing Zhao; Swami, A., "Joint Design and Separation Principle for Opportunistic Spectrum Access in the Presence of Sensing Errors," IEEE Transactions on Information Theory, Vol 54, Issue 5, pp. 2053 - 2071, May 2008

[6] I. F. Akyildiz, W. Y. Lee, M. C. Vuran, and S. Mohanty, "NeXt generation/ dynamic spectrum access / cognitive radio wireless networks: A survey," Computer Networks Journal (Elsevier), vol. 50, no. 13, pp. 2127-2159, September 2006

[7] J. So and N. H. Vaidya, "Multi-channel MAC for ad hoc networks: handling multi-channel hidden terminals using a single transceiver," in Proc. 5th ACM International Symposium on Mobile ad hoc Networking and Computing, Roppongi Hills, Tokyo, Japan, May 2004, pp. 222-233

[8] I. F. Akyildiz, W. Y. Lee, M. C. Vuran, and S. Mohanty, “A survey on spectrum management in cognitive radio networks," IEEE Commun. Mag., vol. 46, no. 4, pp. 40-48, April 2008

[9] J. Jia, Q. Zhang, and X. Shen, "HC-MAC: a hardware-constrained cognitive MAC for efFcient spectrum management," IEEE J. Sel. Areas Commun., vol. 26, no. 1, pp. 106-117, 2008

[10] H. Su and X. Zhang, "Cross-Layer Based Opportunistic MAC Protocols for QoS Provisionings Over Cognitive Radio Wireless Networks," IEEE J. Sel. Areas Commun., vol. 26, no. 1, pp. 118-129, January 2008

[11] M. M. Buddhikot, P. Kolodzy, S. Miller, K. Ryan, and J. Evans, "DIMSUMNet: New Directions in Wireless Networking Using Coordinated Dynamic Spectrum Access," in WOWMOM '05: Proc. Sixth IEEE International Symposium on World of Wireless Mobile and Multimedia Networks, 2005, pp. 78-85

[12] J. P. Romero, O. Sallent, R. Agusti, and L. Giupponi, “A novel on demand cognitive pilot channel enabling dynamic spectrum allocation," in 2nd IEEE International Symposium on New Frontiers in Dynamic Spectrum Access Networks, (DySPAN'07), April 2007, pp. 46-54

[13] T. C. Clancy, "Dynamic spectrum access using the interference temperature model," Annals of Telecommunications, Springer, vol. 64, no. 7, pp. 573-592, 2009

[14] C. Cordeiro, K. Challapali, and M. Ghosh, "Cognitive PHY and MAC layers for dynamic spectrum access and sharing of TV bands," in Proc. first international workshop on Technology and policy for accessing spectrum (TAPAS'06), Boston, MA, USA, August 2006

[15] C. Cordeiro and K. Challapali, "C-MAC: A cognitive MAC protocol for multichannel wireless networks," in Proc. Symposium on Dynamic Spectrum Access Networks (DySPAN'07), April 2007, pp. 147-157

[16] K. Ghaboosi, M. Latva-aho, and Y. Xiao, "A Distributed Multi-channel Cognitive MAC Protocol for IEEE 802.11s Wireless Mesh Networks," in 3rd International Conference on Cognitive Radio Oriented Wireless Networks and Communications (CrownCom'08), May 2008, pp. 1-8

[17] L. Ma, X. Han, and C. C. Shen, "Dynamic open spectrum 
sharing MAC protocol for wireless ad hoc networks," in Proc. Symposium on Dynamic Spectrum Access Networks (DySPAN'05), Baltimore, MD, USA, November 2005, pp. 203-213

[18] T. Chen, H. Zhang, G. M. Maggio, and I. Chlamtac, "Cogmesh: A cluster-based cognitive radio network," in Proc. Symposium on Dynamic Spectrum Access Networks (DySPAN'07), Dublin, Ireland, November 2007, pp.

\section{$168-178$}

[19] M. Timmers, S. Pollin, A. Dejonghe, L. Van der Perre, and F. Catthoor, "A Distributed Multichannel MAC Protocol for Multihop Cognitive Radio Networks," IEEE Trans. Veh. Technol., vol. 59, no. 1, pp. 446-459, January 2010 